\title{
Managing Flexibility: Modeling Binding-Times in Simulink
}

\author{
Danilo Beuche ${ }^{1}$ and Jens Weiland ${ }^{2}$ \\ 1 pure-systems GmbH, D-39106 Magdeburg, Germany \\ danilo.beuche@pure-systems.com \\ 2 Reutlingen University, Department of Technology, D-72762 Reutlingen, Germany \\ jens.weiland@reutlingen-university.de
}

\begin{abstract}
Model-based development is supposed to improve the development efficiency by raising the abstraction level and generating applications instead of manually coding the application in low level languages like C. One of the successful incarnations of this idea is the MATLAB Simulink tool chain. These tools are now widely used in the automotive industry not only to simulate control devices but also to generate product quality code from it. Like with traditional concepts reuse of created models is an issue. When this can be done efficiently, an additional level of effort reduction (and quality improvement) will be achieved. While MATLAB Simulink in combination with code generators provides good support for creating models for single application, and libraries of models, it does not provide sufficient support for more complex reuse scenarios with fine grained variations across the model(s). This paper will extend the approach developed by an automotive car manufacturer to address these issues. After a discussion of the basic concept the paper will put a special focus on support for flexible binding times since this is one of the crucial issues for reusing models across different projects with different need for run-time switchable variations (development/testing) and static decisions for product generation (resource efficiency in terms of code size and run-time). A concrete application supporting the deployment of these concepts in a project, developing mass production control units, is discussed in the following section.
\end{abstract}

\section{Introduction}

While in its early days, the automotive industry produced relatively simple cars with not much variability, like the Ford Model T, todays cars are highly configurable and provide quite a lot of variability. Due to the huge amount of vehicle configurations and varying requirements related to differences in hardware, regulatory, and market behavior there are many aspects of this variability [14. Today embedded software plays a central role since most functions in a car are software-based. Thus, functional variability leads directly to variability in the embedded software. Development of embedded software is increasingly done model-based, using code generators to produce production code directly from 
models. Mathworks Matlab tool chain with its extensions Simulink and Stateflow is an important exponent used in the automotive industry. The graphical modeling languages enable developers to specify, model, and simulate signal flow oriented and state based systems. The implementation can be done using code generators like The Mathworks Realtime Workshop Embedded Coder or dSpaces TargetLink. Simulink models are basically signal flow graphs composed of elementary blocks and state charts. Complex subfunctions can be represented as sub-system blocks, which internally are again signal flow graphs. Signal connections represent data that flows between model blocks while simulating the model. Simulink provides a large set of blocks for functions like logical operations or signal routing. Each block has a set of parameters, which allow the configuration of the blocks behavior, properties, and its presentation. Code generators like TargetLink add additional parameters to maintain code generation related information. To properly deal with variability in the automotive context, an economic view on reuse of specifications, models, architectures, components, or even documentation and tests, is essential. Model-based software development, with a holistic use of models in all phases of development including abstraction from concrete target platforms, is an important step towards improved reuse. However, when it comes to systematic consideration of variability (e.g. variable data or functions), additional concepts are a necessity. Especially, when applying model-based development using Simulink and Stateflow, deficits with respect to variability handling become obvious:

- There are only insufficient description capabilities for modeling of structural variability in Simulink. This variability cannot be described unambiguously, and process safe. Process safe in this context means that in every phase of software development variability and its implications can be traced unambiguously.

- Due to missing variability handling concepts in Simulink checking of model configurations in order to detect invalid variability configurations is not possible.

6 describes basic concepts for systematic modeling and configuration of variability in Simulink, used to develop automotive software. The article presented here goes further and discusses a concept for implementing flexible binding time support. Binding times specify the point in time at which variability is removed and a concrete instantiation of a vehicle function is selected. Depending on the use case, an optional vehicle function may be removed completely, thus, there is no code for it on the vehicles electronic control unit (ECU) at all, or is just turned off using a data parameter, but remains present in the code and can be activated later, e.g. at a service station or at run-time. Selection of binding times has a strong influence on the amount of code present in a vehicle (in general, the later the decision is made, the more code is generated). Flexible selection of binding times permits to control this by choosing for each point of variation the optimal binding time. Due to the high number of produced hardware units even small difference in code size can have a huge impact on production cost of these units. The remainder of this paper describes the approach for flexible 
binding time support based on the concepts from [6. In section 2 basic concepts are presented. Section 3 takes a closer look at binding times, followed by section 4 , which discusses in detail the taken approach for modeling and configuration ofing binding times. The application of these concepts is shown in section 5 . Section 6 provides a summary of the paper. The presented concepts are based on the tool chain Matlab 7.1, TargetLink 2.1 and pure::variants 2.4.

\section{Modeling and Configuring Variability in Simulink}

The concepts for handling variability in Simulink presented in [6] are based on the Generative Software Development Model 44. Based on the engineering of system families its main goal is an automated generation of individual family members. The main element of this concept is the Generative Domain Model. It separates application oriented concepts (problem domain concepts) from the implementation (solution domain concepts). Configuration knowledge is used for mapping both domains onto each other and, thus, it explicitly links them together. To describe the application oriented concepts feature modeling is being used. Feature models provide an abstract representation of variability in a system family on the problem domain level. Feature models contain common and variable features and their dependencies [2/4/12]. Starting point for describing variability in Simulink-models is the variation point (see meta model shown in Fig. 1). The variation point encapsulates variability information, which has to be added to the Simulink model in order to allow systematic handling of variability.

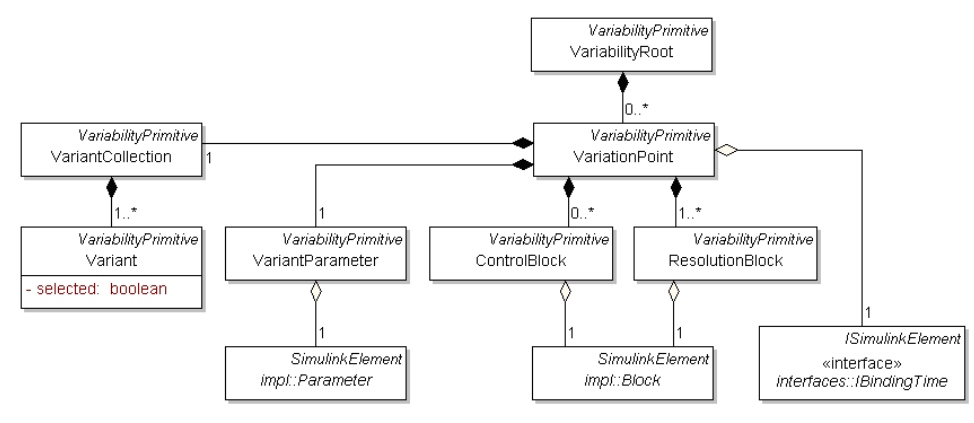

Fig. 1. Variation point meta model

Central element of a variation point is its uniquely identifiable VariantParameter. This parameter is the actual point where functional variants are created by applying different settings to this parameter. The variation point permits only for a finite number of choices. These choices are represented in the VariantCollection and are mapped to unique variant parameter values. According to 9] a variation point identifies one or more locations at which variation will occur.. These locations are internally represented by mechanisms, which remove variability once the variation point has been configured. The block library of 
Simulink provides a number of blocks which can be used to realize variability mechanisms [1015. Examples are:

- Conditionally executed subsystems (Enabled Subsystem-block, Function Call Subsystem-block),

- Signal routing blocks (Switch-block, Multiport Switch-block),

- Logical gates (AND-block, OR-block),

- Configurable subsystems (Configurable Subsystem-block),

$-\ldots$

Each of these blocks has its own way of controlling the variable functionality. E.g. a function encapsulated in an Enabled Subsystem can be (de)activated depending on the value of the Enabled signal sent to this block. Thus, the Enabled Subsystem is well suited for representing optional functionality in the model. In similar ways logical conditions represented by $A N D$ or $O R$ blocks are able to control the execution of optional functionality by controlling the transmission of input signals depending on the setting of a selected input signal. The Switchblock selects a variant based on a Control-signal, similar to the if-else found in programming languages like C. Therefore the Switch-block is well suited especially for modeling alternative functionality. Blocks which resolve variability are called Resolution Blocks in our context. Most of these blocks require an input signal, which controls the execution of the block. To perform the actual selection of a variant a designated Control Block should be used for providing the input signal. This block can be represented by a parameterized Constant-block or a Data Store Read-block. Depending on the value represented by the control block, the respective variant is executed.

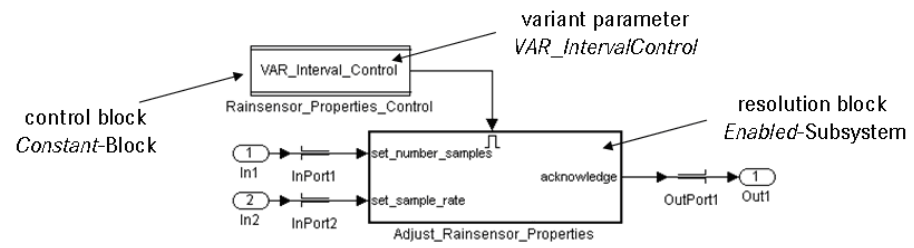

Fig. 2. Variability mechanism example

Fig. 2 shows an Enabled Subsystem whose execution is controlled by a Constant-block. The Constant-block references the workspace parameter VAR_IntervalControl. In our example this parameter represents the VariantParameter. Depending on the value of this parameter one or zero the Enabled Subsystem Adjust_Rainsensor_Properties will be executed or not. The set of control and resolution blocks blocks is called Variant Blocks. To clearly separate these blocks from other Simulink blocks, an additional mask parameter, e.g. VarInfo, is used. Mask parameters extend Simulink-blocks with additional properties. By adding the parameter VarInfo to a block, the regarded block will be treated as variant block. The mask parameter references the variation point, to which the block is related. 


\section{Taking Binding Time into Account}

A variation point is a kind of place holder, which must be filled by a valid variant instance at a certain point during creation of a system variant. The decision, which of the possible instances for variation point to select, is called binding. And consequently the time at which this decision occurs is called binding time. There are different binding time models described in literature ([3811]), there are even specialized models for embedded automotive software [7]. The binding time model used for the approach in this paper is based on the FODA binding time model [1]. It distinguishes between three different binding times:

- CompileTime: The variation point is bound during compilation.

- LoadTime: The variation point is bound when the system starts up, e.g. by reading parameters from databases or from the system environment.

- RunTime: Variation points can be bound at any time during the execution of the system.

Since model based development introduces additional abstraction level at which variation points can be bound, the approach introduces a new binding time:

- ModelConfigurationTime: Variation points are bound when a variant-rich model is instantiated / (in our context configured) to represent a single variant.

In the automotive context most decisions are taken before run-time due to improved performance and resource consumption. There are also regulatory requirements, demanding that certain decisions cannot be changed while the car is running, i.e. the software is being executed. This is somewhat contradictory with the wish to bind the variations as late as possible during the production or even the delivery of the car, , since this would reduce logistic efforts (just one programmed hardware unit for a set of supported engine types vs. differently programmed hardware units for each engine type).

\section{Towards a Binding Time Concept for Simulink Models}

A flexible selection of a binding time for individual variation points adds another dimension of decision since for each variation point there could be one or more binding times at which the decision can be made. The decision, which binding times to offer should be independent from the functional variability in the problem domain. With traditional software approaches based on manually written code, this introduces a huge challenge, since code supporting different binding times will look different. This becomes evident in scenarios where organizations are forced to provide the same functionality with different binding times. An example is the concept of pre-compile time configured components vs. post-build configured components, in C. To provide support for the first binding time (basically compile time) \#define/\#ifdef preprocessor statements are used. 
For post build configuration normal if statements are used. Due to the different semantics of these two concepts more or less separate implementations are necessary. In model-based development such problems can be solved much more efficiently with an integrated binding time model, since the code generator can take the requested binding time into account when generating code from models.

\subsection{Influence of Code Generation on Variability in Simulink Models}

The challenge of introducing binding time support in Simulink results from the original purpose of Simulink as a simulation tool, not intended for generating code. With respect to the functionality of a (simulated) system, there is no need for anything but run-time binding, since this is the most flexible approach and hardware resources such as memory and processing power are readily available. The situation is different for code generation when it comes to production code generation, where the memory and also processing power on the embedded system are a very limited resource. The selection of appropriate variant blocks and their configuration as well as the selection and configuration of the used code generator have a significant impact on the code generated from variant-rich Simulink-models and, thus, on the binding time of variation points. For example variability represented by Configurable Subsystems is controlled by a local parameter of the Configurable Subsystem-block and already resolved on model level, i.e. before code generation. Unselected subsystems are removed before code generation. [5] refer to this as preprocessing of the model. The advantage of this is that a concrete model variant can be expressed graphical using the normal modeling language.

To provide later binding times, not only the chosen model elements play a role, but also how the code generator transforms model elements to source code. Kalix et. al. investigated in [10 the influence of Simulink and Stateflow model elements on code generated by the Real Time Workshop Embedded Coder and TargetLink, two widely used code generators for Simulink and Stateflow. Their idea was to remove variability at code generation time by relying on the optimizations provided by the code generators. These optimizations effectively remove irrelevant parts of the models from the generated source code. Their conclusion was that at the present time the result was a collection of workarounds. [10. The result heavily relies on the cleverness of the code generator. The following paragraph investigates the capabilities of TargetLink available for representation of earlier than run-time binding.

TargetLink, itself, offers an own set of blocks to be used inside Simulink (referred to as TargetLink blocks). Most of these blocks have their equivalent in Simulink, but extend the equivalent Simulink- blocks by additional properties and behavior regarding code generation and optimization. Analogous to Simulink, TargetLink does not have explicit concepts for managing variability.

During code generation variability on model-level is mapped to data and control structures in the target source code language. In case of TargetLink the language is $\mathrm{C}$ and generated constructs include e.g. \#define/\#ifdef and if-else 
statements. The removal of variability, i.e. the binding, is done by assigning values to variables. The declaration of these variables decides at which point in time the variability is removed. TargetLink provides so called Variable Classes for this purpose. Variable classes define the appearance of a variable in the generated code. These variables represent outputs, states, and parameters of a block. Accordingly, Variable Classes has to be specified in a TargetLink block dialog, e.g. for the output of a Constant-block or the threshold of a Switch-block. Each Variable Class has a set of properties, e.g. related to optimization, where the variables of this class are stored, whether their values could be changed during run-time or not and so forth. TargetLink provides a set of predefined classes, but permits definition of new classes as well. Depending on the chosen Variable Class of a model element, this model element is represented e.g. as a constant or as a global variable in the generated source code. In combination with the optimization of the code generator some blocks will not be present at all in the generated code. Fig. 3 shows this for a Constant-block controlling a Switch-block. In the context of variability the Constant-block represents the control block and the Switch-block represents the resolution block.

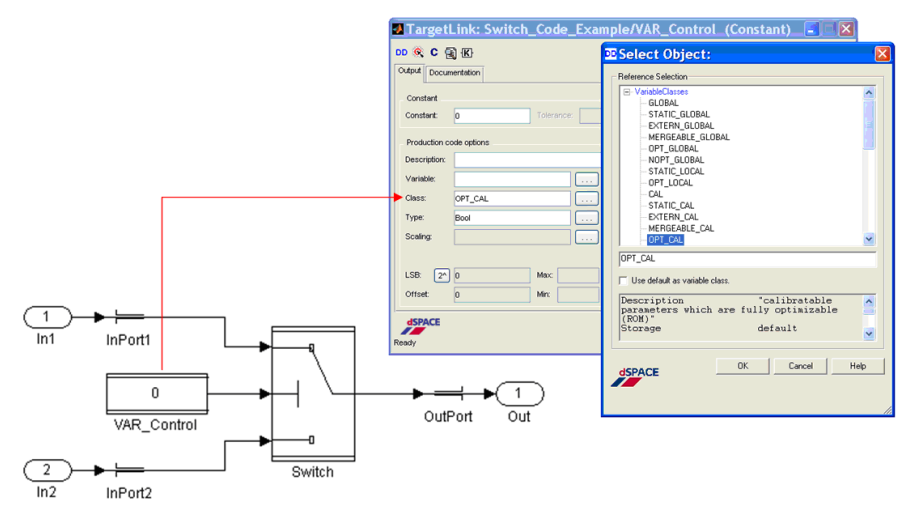

Fig. 3. Signal Routing based on Constant- and Switch-block

Depending on the selection of the Variable Class for the TargetLink Constantblock (in this case OPT_CAL or OPT_LOCAL), the Constant- and Switch-block are present in the generated code or not. Fig. 4 shows the first case, Fig. 5 the later case. Variable Classes with the prefix OPT are predefined classes which allow the code generator to optimize the generated code, i.e., if possible to remove variables out of the source code.

Setting the Variable Class to OPT_CAL tells the generator to declare the variable Sa1_VAR_Control, generated out of the TargetLink Constant-Block block VAR_Control, as const volatile. This makes the variable unchangeable for the generated program but the value might be changed for instance by an interrupt service routine, e.g. for calibration of certain data parameters in the vehicle. The 


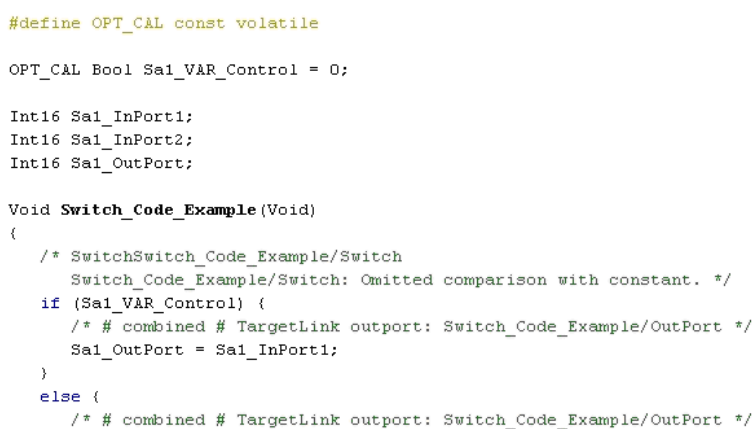

Fig. 4. Extract from source code generated for a Switch-block using Variable Class OPT_CAL

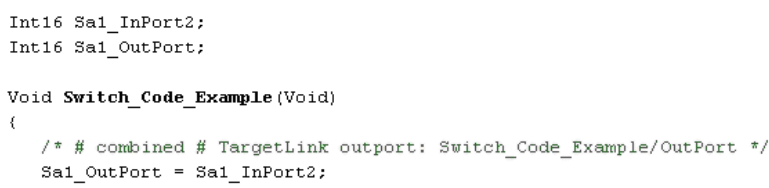

Fig. 5. Extract from source code generated for a Switch-block using Variable Class OPT_LOCAL

code generator must assume for the generated code that the variable could be changed during run-time. Since this change influences the signal routing of the Switch-block, an if-else construct must be generated for the block.

However, if the OPT_LOCAL class is used, the code generator assumes, that the variable will not be changeable by the program (as before), but also knows that it will not be referenced from code not generated from this model. Thus, the code generator can generate code, which uses the knowledge about the setting of the Constant-parameter and uses the right input signal for the out-port (see Fig. 5). There will be no change in the routing during the run-time of the program.

\subsection{Specification of Binding Times for Variation Points in Simulink-Models}

The following concept is based on the assumption that variability not resolved on model level is mapped to variables in the source code by the code generator. These variables are either used as data assignments to adapt behavior of an algorithm or to change the control flow in the application. In case of blocks directly provided by TargetLink it is possible to set the Variable Class declaration of blocks according to the desired binding time explicitly.

From Simulink blocks the code generator uses the context it sees at the time of code generation to decide how to generate optimal code. These Simulink blocks contain either additional TargetLink blocks (e.g. in Fig. 3 a TargetLink 
Constant- and Switch-block), from which code is generated regarding the configuration of their associated Variable Classes, or they are explicitly controlled by TargetLink blocks. This means that for every mechanism, which removes variability in Simulink, an appropriate TargetLink block is available, which can be used to specify a binding time.

In order to allow modeling of binding times an additional mask parameter VarBinding is introduced to complement the VarInfo parameter for all blocks where binding times shall be specifiable. In the context of code generation, this parameter is especially relevant for TargetLink blocks, which express variability in the Simulink model. The content of the parameter VarBinding is represented in form of a structure.

As shown in the example in Fig. 6, besides the binding time specification itself (here: CompileTime), some additional information is stored, e.g. the model elements of the specific TargetLink block, which have Variable Classes assigned. Another important information is whether the binding time might be changed during model configuration (Alterable set to true). For blocks such as the Configurable Subsystem-block which only provides binding at ModelConfiguration Time, the value Alterable is set to false.

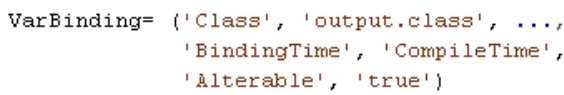

Fig. 6. Example for the data store in a VarBinding parameter

In order to map the available binding times (e.g. ModelConfigurationTime, CompileTime, LoadTime, or RunTime) onto the expected code representation, Variable Classes, representing the equivalent binding time, has to be defined and configured. As part of the model configuration, the setting for binding time as defined in the VarBinding parameter is mapped to the equivalent Variable Class. Part of this process is also validation of the provided information against consistency rules, e.g. that all Control blocks referencing the same VariantParameter may only control resolution blocks which allow the selected binding time.

\section{Application of the Binding Time Concept as Part of the Simulink Configurator}

The approach described in [6] permits systematic modeling, management and configuration of variability in variant-rich Simulink models. The extension for handling binding time described in this paper provides improved optimization for resource usage and performance of code generated for configured model variants. The original implementation of the Simulink Configurator based on MATLAB 7.1, TargetLink 2.1 and pure::variants 2.4 was extended to support the presented binding time concepts. The Simulink Configurator consist of two main parts, a set of blocks for modeling variability in Simulink, a set of dialogs integrated 


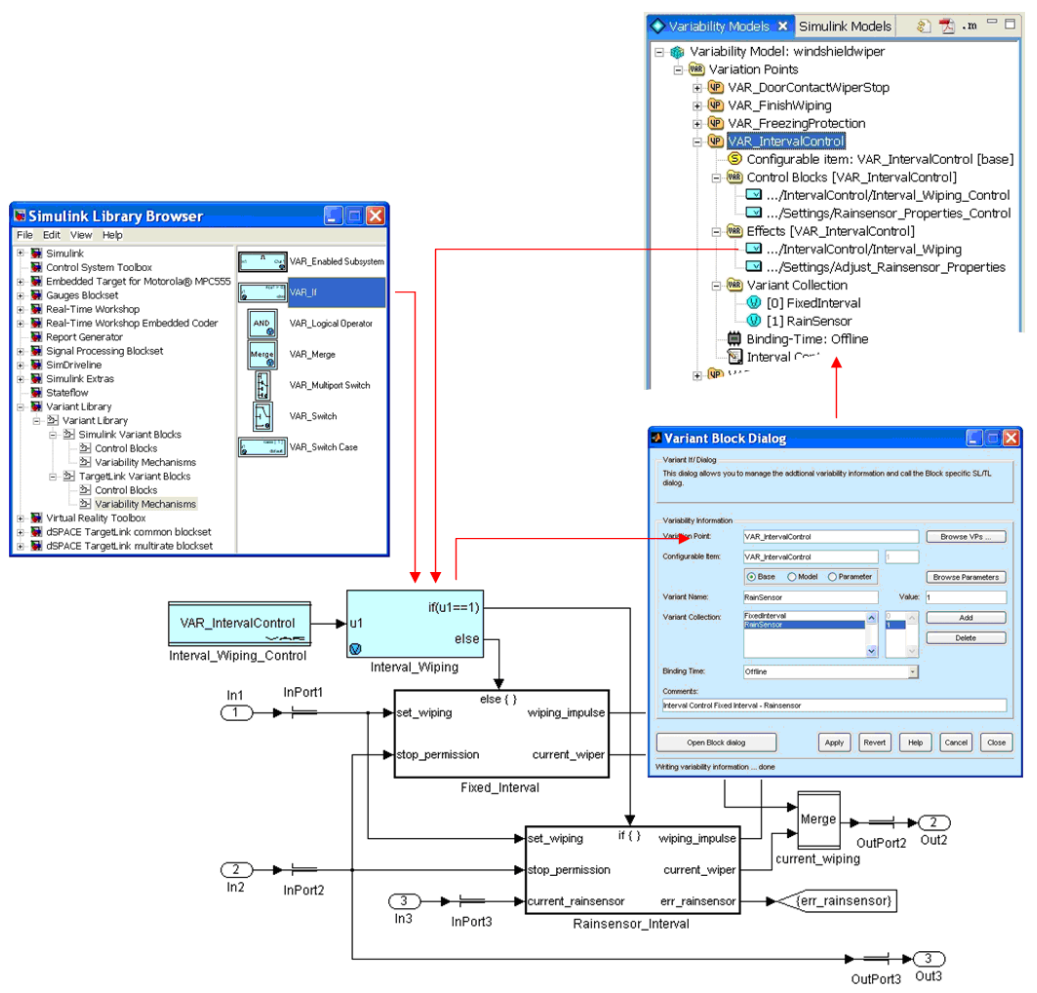

Fig. 7. Design of variable functions in Simulink

into Simulink and a configuration application including feature modeling support based on pure::variants. Fig. 7 shows the basic elements of this tool chain including the support for binding time specification. The workflow is as follows: The model developer selects variant blocks from the library (Fig 7. upper left corner) and inserts those blocks into a Simulink model (Fig 7. lower left corner). These blocks are Simulink- and TargetLink blocks with the additional property and behavior for modeling variability. Mainly, these blocks contain the two mask parameters VarInfo and VarBinding, with their associated information. Afterwards the model developer links the variant blocks to variation points. The definition of new variation points is done using a variant block dialog inside Simulink (Fig 7. lower right corner). Here the binding time support is just a field allowing the specification of a default binding time. The permitted values are Offline (corresponding to CompileTime), StartUp and Online (aka Runtime). The integration of binding time concepts into the existing Simulink Configurator tool chain was straightforward, since it basically links already existing functionalities together. 
The pure::variants based configuration application reads the information stored in Simulink (Fig 7. upper right corner) to permit the linkage of these variation points to features and to describe dependencies between variation points. The model configuration including the specification of variant specific binding times for variation points itself is done in this application. The validity of the selected feature combination is checked and mapped to the corresponding setting for VariantParameters in Simulink and applied to the Simulink model in real-time.

\section{Summary}

The presented concept for binding time specification extends the concepts for formal description, and systematic modeling, and configuration of variant rich systems based on Simulink. The implementation of these concepts in the Simulink Configurator is based on MATLAB, TargetLink and pure::variants functionality. The Simulink- configurator proved to work on real-world problems, since the tool chain is being used in a project for production code development for Mercedes passenger cars.

With the addition of binding time support the Simulink-configurator is able to extend the reach of model reuse beyond individual project. The easily changeable binding time permits flexible configurations for developing and testing of systems based on function-rich models, the scaling down to individual variant instances, and for production code, meeting the resource constraints of final product hardware. While the implementation of the binding time concept in its current form is based on a specific code generator (TargetLink), on variability level it can be ported to other code generators as well since most of the information is stored in an abstract form only and later mapped internal to the concrete mechanism provided by TargetLink. With a standardization of variability information within Simulink, applicable to all blocks (not just the ones from one code generator), it would be possible to flexibly exchange between code generators depending on the use case (e.g. when generating for different target systems or interface standards like AUTOSAR). This is one of the benefits of raising the abstraction level for variability modeling from a rather informal level to a systematic and formal level providing clear separation of concerns.

There are also open issues left. While code generators provide the necessary functions to simulate a binding time concept it would be a much more reliable approach if code generators would include a direct and simple way to control the code generation in this respect. Another issue for further research is the efficient mapping of binding times to appropriate code patterns, which provide traceability and optimal performance.

\section{References}

1. AUTOSAR (2009), http://www.autosar.org

2. Beuche, D.: Composition and Construction of Embedded Software Families. Dissertation, Universität Magdeburg (2003) 
3. Bayer, J., Forster, T., Kiebusch, S., Lehner, T., Ocampo, A., Weiland, J.: Feature- und Entscheidungsmodell-basierte Varianteninstanziierung im PESOAProzess. (engl.: Feature- and Decision-Model-based Variant Instantiation within the PESOA-process). PESOA-Report Nr. 21/2005 (2005)

4. Czarnecki, K., Eisenecker, U.W.: Generative Programming- Methods, Tools, and Applications. Addison-Wesley, Reading (2000)

5. Creutzburg, U., Kalix, E.: Process Integration of Model-Based Design and Production-Code Generation in the Multi-User / Multi-Project Development Environment at Continental Teves- Part 2. In: Proceedings of the Int. Automotive Conference (2004)

6. Dziobek, C., Loew, J., Przystas, W., Weiland, J.: Von Vielfalt und Variabilität Hand-habung von Funktionsvarianten in Simulink-Modellen. (engl.: Model Diversity and Variability - Handling of Functional Variants in Simulink-Models). Elektronik automotive (February 2008)

7. Fritsch, C., Lehn, A., Strohm, T.: Evaluating Variability Implementation Mechanisms. In: Proceedings of the 2nd Int. Workshop on Product Line Engineering The Early Steps (PLEES 2002), Seattle, USA (2002)

8. Geyer, L.: Variabilitätsmanagement in Produktfamilien (engl.: Variability Management in Product Families). Dissertation, Universität Kaiserslautern (2003)

9. Jacobson, I., Griss, M., Jonsson, P.: Software Reuse- Architecture, Process, and Organization for Business Success. Addison-Wesley, Reading (1997)

10. Kalix, E., Bunzel, S., Judaschke, U.: Variant Coding in Model-Based Design. In: 9th World Multiconference on Systemics, Cybernetics, and Informatics (WMSCI), Orlando, USA (2005)

11. Kang, K.C., Cohen, S.G., Hess, J.A., Nowak, W.E., Peterson, A.S.: Feature Oriented Domain Analysis (FODA) Feasibility Study. Technical Report CMU/SEI90-TR-21, Carnegie Mellon University, Pittsburgh, PA (1990)

12. Lee, K., Kang, K.C., Lee, J.: Concepts and Guidelines of Feature Modeling for Product Line Software Engineering. In: Gacek, C. (ed.) ICSR 2002. LNCS, vol. 2319, p. 62. Springer, Heidelberg (2002)

13. pure-systems, pure:variants Eclipse Plugin User Guide (2008)

14. Schäuffele, J., Zurawka, T.: Automotive Software Engineering - Grundlagen, Prozesse, Methoden und Werkzeuge (engl.: Automotive Software Engineering Foundations, Processes, Methods, and Tools) Vieweg, Wiesbaden (July 2003)

15. dSpace GmbH: TargetLink Advanced Practices Guide - for TargetLink 2.2. dSpace, Paderborn (2006) 